\title{
ON THE APPLICATION OF PIEZOLAMINATED COMPOSITES TO DIAPHRAGM MICROPUMPS
}

\author{
Raffaele Ardito ${ }^{*}$, Emanuele Bertarelli ${ }^{*}$, Alberto Corigliano ${ }^{*}$, Giacomo Gafforelli \\ * Department of Structural Engineering \\ Politecnico di Milano \\ Piazza Leonardo da Vinci 32, 20133 Milan, Italy
}

e-mail: \{ardito, bertarelli, coriglia, gafforelli\}@stru.polimi.it, web page: http://www.stru.polimi.it/mems

Key words: Micropump, Plate model, Finite Element analysis, Piezoelectric materials.

Summary. This paper deals with the numerical simulation of piezolaminated microplates adopted as actuators in micropumps. The performances of piezoelectric actuation is critically assessed by means of comparisons with devices based on the electrostatic force.

\section{INTRODUCTION}

Silicon micropumps [1] are the "heart" of active microfluidic systems that allow one to perform precise manipulation of fluids for many applications.

Figure 1 shows the scheme of a micropump, with the active diaphragm, represented by a circular microplate clamped all along the boundary (red element) and the inlet and outlet valves. This research is focused on the actuation technique for the diaphragm. In recent times, the Authors [2] have studied the possible application of electrostatic forces in order to actuate the circular microplate: the vertical deflection of the plate is obtained by imposing a voltage difference between the plate itself and the substrate. In this paper, the possible application of piezoelectric actuation is examined: consequently, the plate is represented by a laminated composite with alternated silicon and piezoelectric layers.

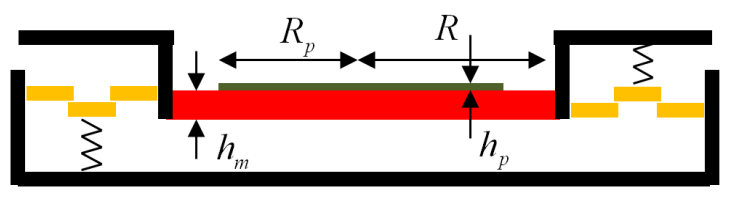

Figure 1: Scheme of the micropump.

\section{MODELING ISSUES AND MAIN RESULTS}

The numerical analyses have been focused on the quasi-static response of the plate. To this purpose, a Mindlin-Reissner model has been adopted. The sectional behavior of the plate is evaluated by means of the Classical Laminated Theory (CLT), modified in order to include the piezoelectric constitutive model [3]. The latter has been simplified by neglecting the socalled "direct effect", namely the creation of electric current due to mechanical deformation. The "indirect effect" is represented by a planar strain induced by the current flowing in the 
vertical direction. Such an applied strain, referred to an asymmetric piezoelectric layer (green element in Figure 1), yields the vertical deflection of the plate.

The quasi-static problem has been solved by means of a Matlab-based Finite Element program. Given the geometric features (shown in Figure 1) and the applied voltage across the piezoelectric layer, the plate deflection has been evaluated. In the post-processing phase, it has been possible to compute the stroke volume (namely, the variation of the pumping chamber volume after the plate deflection) and the necessary power. The maximum stroke volume is obtained if $R_{p} / R=0.73$. Figure 2 shows the comparison between electrostatic and piezoelectric actuation. It is possible to conclude that the piezoelectric actuation is less expensive than the electrostatic one only for very small stroke volumes; on the other hand, the required voltage is always smaller. The latter parameter is of great importance in view of possible biomedical applications, which impose a strict limitation on the applied voltage.

\section{FUTURE PROSPECTS}

The quasi-static analyses have been complemented by dynamic analyses, in the absence of fluid. In this way, it has been possible to obtain preliminary information on the natural frequency of the plate and on its behavior for various levels of damping. The next step will be represented by fully coupled fluid-dynamic analyses, which will provide conclusive data on the pumping efficiency for different actuation techniques.
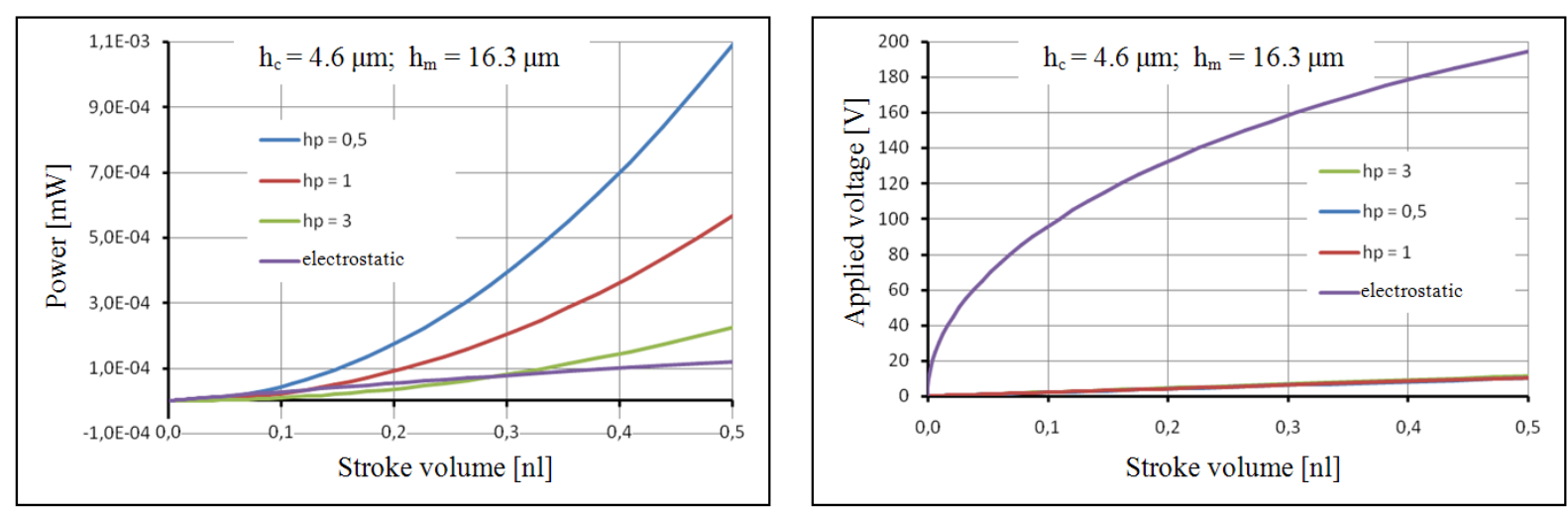

Figure 2: Piezoelectric vs. electrostatic actuation: comparison of dissipated power and applied voltage (hc represents, in the electrostatic case, the capacitor gap i.e. the distance between the plate and the substrate).

\section{REFERENCES}

[1] B. Iverson and S. Garimella, "Recent advances in microscale pumping”, Microfluid. Nanofluid., 5, 145-174 (2008).

[2] E. Bertarelli, R. Ardito, R. Contro and A. Corigliano, “A plate model for the evaluation of pull-in instability occurrence in electrostatic micropump diaphragms”, Int. J. Appl. Mech., 3, 1-19 (2011).

[3] K.G. Webber, D.P. Hopkinson and C.S. Lynch, "Application of a classical lamination theory model to the design of piezoelectric composite actuators", J. Intell. Mat. Syst. and Struct., 17, 29-34 (2006). 